\title{
thumanidades
}

Revista Humanidades

ISSN: 2215-3934

humanidades@ucr.ac.cr

Universidad de Costa Rica

Costa Rica

\section{Desmitificando al ser humano desde la Economía Conductual}

Pérez Martínez, Dr. Armenio; Rodríguez Fernández, Dra. Aimara

Desmitificando al ser humano desde la Economía Conductual

Revista Humanidades, vol. 12, núm. 1, e49301, 2022

Universidad de Costa Rica, Costa Rica

Disponible en: https://www.redalyc.org/articulo.oa?id=498068490015

DOI: https://doi.org/10.15517/h.v12i1.49301

\section{(c) $(1) \Theta$}

Esta obra está bajo una Licencia Creative Commons Atribución-NoComercial-SinDerivar 3.0 Internacional. 
Desde las ciencias sociales, filosofía y educación

\title{
Desmitificando al ser humano desde la Economía Conductual
}

\author{
Demystifying the Human Species from Behavioral Economy \\ Dr. Armenio Pérez Martínez \\ Universidad Laica Vicente Rocafuerte, Guayaquil, Ecuador \\ aperezm@ulvr.edu.ec \\ iD https://orcid.org/0000-0002-9491-6938 \\ Dra. Aimara Rodríguez Fernández \\ Universidad Laica Vicente Rocafuerte, Guayaquil, Ecuador \\ airodriguezf@ulvr.edu.ec \\ iD https://orcid.org/http:// \\ orcid.org/0000-0002-6654-2588
}

DOI: https://doi.org/10.15517/h.v12i1.49301

Redalyc: https://www.redalyc.org/articulo.oa?

$\mathrm{id}=498068490015$

Recepción: 12 Octubre 2021

Aprobación: 19 Noviembre 2021

\section{Resumen:}

Los aportes de la Economía Conductual contribuyen a desmitificar estas declaraciones que justifican determinados comportamientos discriminatorios del ser humano. Este artículo tiene como objetivo analizar los criterios de la Economía Conductual que contribuyen a desmitificar la esencia humana y sus comportamientos nocivos para el desarrollo de la vida en el planeta. Se realiza una investigación teórica, a partir de la selección de obras clásicas de los últimos 50 años que abordan el tema objeto de estudio, aplicando métodos teóricos como el análisis y la síntesis. Se concluye que la racionalidad humana es limitada, la información se percibe de manera parcial, se analiza de modo incompleto y las decisiones están influenciadas por los heurísticos y sesgos cognitivos, además de las emociones y estados de ánimo de las personas. De manera que se desmitifica las posturas de superioridad hegemónica del ser humano en la naturaleza y del hombre sobre la mujer.

Palabras Clave: Evolución, especie humana, comportamiento, comportamiento económico.

\section{AbStraCt:}

The contributions of the Behavioral Economy contribute to demystify these statements that justify certain discriminatory behaviors of the human being. This article aims to analyze the Behavioral Economy criteria that contribute to demystifying the human essence and its harmful behaviors for the development of life on the planet. A theoretical investigation is carried out, based on the selection of classic works from the last 50 years that address the subject under study, applying theoretical methods such as analysis and synthesis. It is concluded that human rationality is limited, information is partially perceived, incompletely analyzed, and decisions are influenced by heuristics and cognitive biases, in addition to people's emotions and moods. Thus, the positions of hegemonic superiority of the human being in nature and of man over woman are demystified.

KEYWORDS: Evolution, human Species, behavior, economics behaviour.

\section{INTRODUCCIÓN}

La existencia de la especie humana está en juego. En este sentido, las condiciones necesarias para la vida en el planeta Tierra se han deteriorado en tales proporciones que la posibilidad de que futuras generaciones gocen de los beneficios de la naturaleza es escasa, además el crecimiento económico impuesto por el capitalismo entre los siglos XIX al XXI ha ido consumiendo todas las riquezas ambientales (Ávila-García, 2016; Paz, 2016; Ros, 2019).

Unido a esto, se fueron generando un conjunto de condiciones políticas y sociales para perpetuar el hambre, el analfabetismo, la insalubridad, la escasez de agua, la mala calidad del aire, la pobreza, la desigualdad económica, entre otras calamidades (OXFAM Internacional, 2021). Lastimosamente, las prácticas humanas 
asociadas a estos modelos de producción y consumo perjudiciales han encontrado justificación científica en aras del desarrollo económico desde múltiples ciencias sociales, particularmente la Economía. Este hecho es preocupante, ya que no existe una postura crítica de las prácticas tendientes a excluir las condiciones para la vida en el planeta (Villalobos, Ramírez y Díaz-Cid, 2019).

La Economía es una ciencia social en pleno desarrollo académico. Su impulso se debe al creciente interés por el bienestar humano y la forma de disminuir las brechas económicas y sociales, para generar mejores condiciones de vida. Desde su surgimiento, en el siglo XVIII, ha estado presente acompañando el desarrollo social de la especie humana. Aunque existe aceptación acerca del surgimiento de la Economía como ciencia en 1776, por el papel científico y las obras de Adam Smith, las raíces del pensamiento económico empírico se pierden en los orígenes de la evolución humana.

La Economía ha desarrollado un cuerpo teórico y metodológico influenciado por el paradigma dominante en las ciencias, como ha ocurrido con otras ciencias sociales. Su surgimiento dio lugar a una diversidad de interpretaciones del objeto de estudio que investigaban, generando un conocimiento que se construía desde la pluralidad de experiencias e investigaciones. El estudio de la producción, distribución, cambio, consumo, escasez, precios, mercado, entre otras categorías, han marcado esta variedad de enfoques enriquecedora de la ciencia económica (Etxezarreta, 2015). La postura defensiva de la racionalidad humana imperaba en todas las escuelas económicas, desconociendo los avances de las ciencias psicológicas al estudiar el carácter complejo de las decisiones y la diversidad humana.

Todos los aportes realizados por la Economía convencional han posibilitado la construcción de un arquetipo de sujeto, actor, agente u Homo Economicus, como se le conoce popularmente. Las consideraciones sobre el funcionamiento de este agente económico han sido generalizadas a toda la sociedad y sus individuos, lo que ha creado posiciones académicas marcadas por el absolutismo, el reduccionismo y la grandilocuencia.

La Economía Conductual es un área de investigación científica dentro de las ciencias económicas que rompe con la tradición de concebir a un ser humano individualista, maximizador de beneficios, capaz de analizar el entorno y estructurado en sus decisiones. Sus principales avances científicos se desarrollan a partir de la mitad del siglo XX, esto le permitió obtener reconocimiento por su influencia en la política pública y en el ámbito académico (Simon, 1972; Kahneman, 2017; Sunstein y Thaler, 2017; Rampello, 2019). Las últimas dos décadas han marcado la mayoría de edad para la Economía Conductual por varias razones. En primer lugar, el reconocimiento en el mundo científico como son la obtención de dos premios Nobel de Economía. En segundo lugar, el aumento de las publicaciones académicas en revistas científicas de alto impacto; el acceso al financiamiento de varios organismos internacionales y Estados; así como por la aplicación en las políticas públicas y asesoramiento a importantes decisiones gubernamentales.

Los aportes del conocimiento generado desde la Economía Conductual tienen que encontrar espacio dentro de otras ciencias sociales, influyendo en la visión y perspectivas del ser humano. El papel que desempeñan estos hallazgos científicos permean la concepción del ser humano imperante y que ha estado confirmada por la Economía convencional. La existencia de las personas y la manera en que son socialmente reconocidas depende de su comportamiento y las decisiones que toman, actuando de manera transformadora sobre sus condiciones de vida. Siendo esta una verdad de Perogrullo, las personas se encuentran marcadas por sus acciones y las decisiones que las determinan.

Los mitos y creencias han formado parte de la evolución humana, al punto de ser una forma válida de comprender el modo de dominar grandes grupos, sobre todo, existió la colaboración y la cooperación para producir y obtener soluciones a los distintos problemas que enfrentó la especie humana. Sin embargo, también sirven para justificar determinadas prácticas nocivas para el desarrollo de la vida de las demás especies de la naturaleza (Noah, 2014, 2016, 2018; Sachs, 2008). El conocimiento científico ha influido notablemente en los criterios hegemónicos de la especie humana sobre los demás seres vivos; así como en el criterio de singularidad y diferenciación en cuanto al género. 
De esta manera, este artículo brinda un aporte académico para una concepción novedosa del ser humano. Así, se posibilita una interpretación explicativa e integradora desde la Economía, la Psicología y la Antropología, amparados en reflexiones realizadas desde la Economía Conductual, las cuales ofrecen explicaciones académicas para refutar determinados mitos que existen sobre el ser humano. El objetivo propuesto en este artículo es analizar los criterios de la Economía Conductual que contribuyen a desmitificar la esencia humana y sus comportamientos nocivos para el desarrollo de la vida en el planeta.

\section{METODOLOGÍA}

Desde la perspectiva metodológica, se utilizó el paradigma cualitativo para la selección e interpretación de la información científica, asumiendo una postura crítica y constructiva del conocimiento; determinando unidades de análisis significativas como son la complejidad; la singularidad y el enfoque de género. La investigación realizada es de tipo teórica, por el manejo de la información realizado y por el empleo de métodos del nivel teórico, tales como el análisis, la síntesis y la contrastación teórica (Taylor y Bogan, 1987; Miller, 2006).

A lo largo de la investigación predominó el empleo del método de análisis documental. Este método permitió detectar, obtener, consultar y analizar la bibliografía de manera selectiva, lo que fue útil para los fines propuestos por el investigadores (Hernández, Fernández y Baptista, 2014). Asimismo, se consultaron fuentes primarias. Se desarrolló la investigación a partir de los siguientes procedimientos:

- Búsqueda de fuentes primarias: la recabación de información se realizó a partir de la identificación de materiales académicos relevantes por su impacto en las principales bases de información científica.

- Las principales obras consultados son de Daniel Kahneman, Richard Thaler, Cass Sunstein, Herbert Simon, George Katona y Yuval Noah. Estos autores gozan de reconocido prestigio en el ámbito académico de las ciencias sociales.

- Lectura de los documentos seleccionados: revisión de los documentos seleccionados para establecer las unidades de análisis.

- Recolección de datos: disposición de los datos e información según las unidades de análisis.

- Análisis de la información recolectada: valoración de la información y reflexiones en cada unidad de análisis.

- Redacción del artículo científico: con posterioridad, se presentan los resultados, a partir de la generalización de los hallazgos teóricos encontrados. Eso permite aportar nuevas perspectivas de análisis y considera los criterios de la Economía Conductual.

\section{ANTECEDENTES TEÓRICOS}

Para comprender las posturas actuales sobre el ser humano, es necesario revisar los antecedentes científicos que han fomentado las ideas de superioridad sobre las demás especies, y la diferenciación de capacidades basado en el género. La concepción de Homo Economicus es el resultado de la aplicación en la Economía, de la teoría de la Elección Racional de las Ciencias Sociales (Quintana y Galarza, 2017) y el paradigma utilitarista de la Filosofía (Bentham, 1948; Cejudo, 2019). A partir de la aceptación del supuesto de la racionalidad ilimitada se considera que ha sido distorsionada del ser humano y sus relaciones con el entorno.

El funcionamiento de la sociedad es inconcebible sin la aplicación del conocimiento económico, a pesar de que seamos conscientes de que existen pocos acuerdos y criterios homogéneos; aun viendo lo parcelado del territorio que se pretende analizar desde la Economía, lo intrincado y particular de su objeto de estudio, así como su diversidad metodológica. Se ha puesto en evidencia la incapacidad del paradigma económico para 
dar respuesta a los problemas sociales derivados del uso y creación de bienes (García-Andrés, 2016; Pérez y Rodríguez, 2020).

Como reconoce Tirole (2017), la Economía fue la primera ciencia social que aspiró y consiguió el reconocimiento de su carácter científico, al estar estrechamente relacionada con la Estadística, la Matemática, el pensamiento positivista y el reduccionismo metodológico. La Economía enfatizó el carácter objetivo de sus investigaciones, por lo que la subjetividad de los individuos y del investigador es suprimida, lo que la convierte en una ciencia objetiva y neutral, según las pretensiones de sus primeros investigadores.

En 1932, Lionel Robbins publica su obra Ensayo sobre la naturaleza y la importancia de la Ciencia Económica, en la cual aborda importantes criterios que hoy persisten sobre la Economía. Para este autor la Economía:

es la ciencia que trata de aquel aspecto de la conducta humana que surge de la escasez de medios para conseguir fines dados. (...) La Economía es la ciencia que estudia la conducta humana como una relación entre fines y medios limitados que tienen diversa aplicación (citado en García-Rubio, 2016, p. 34).

La Economía convencional evidencia una visión centrada en el agente económico, sin interés por el estudio de la naturaleza y el uso de sus recursos. Este conocimiento aísla al individuo a la hora de realizar el análisis del hecho económico, con lo que se genera una posición de superioridad y dominio. La Economía ha dado un punto de vista de muchos objetos, a merced de la voluntad y las necesidades de la especie humana, la cual dispone de los recursos del mar, de la tierra, del aire. De hecho, en muchos casos los transforma para consumirlos o almacenarlos, generando posesión sobre ellos (Von Mises, 1980; Sachs, 2008).

Se pueden resumir las principales características de la Economía convencional a partir de los siguientes criterios: "epistemología instrumentalista, el individualismo metodológico, una racionalidad ilimitada y una concepción de la economía centrada en la escasez y los intercambios” (García-Rubio, 2016, p. 46). En consecuencia, la ciencia económica ha acumulado, por más de 250 años, conocimiento suficiente para respaldar los siguientes mitos y creencias (Allais, 1953; Angrist y Pischke, 2015; Calvo, 2018; DellaVigna, 2009; Etxezarreta, 2015; García-Andrés, 2016; Pérez y Rodríguez, 2020):

- La capacidad humana de alcanzar siempre el mayor beneficio o utilidad posible.

- La capacidad humana de analizar objetivamente las condiciones concretas para el desarrollo de la vida en sociedad.

- La capacidad humana de mantener preferencias, elecciones y comportamientos claros prácticamente inmutables.

- La posibilidad permanente del ser humano de satisfacer todas sus necesidades de manera ilimitada (Principio del carácter creciente de las necesidades humanas). Considera a la necesidad humana como motor de las aspiraciones y el desarrollo social.

- El uso ilimitado de los recursos naturales.

- Los mecanismos económicos cooperativos a la hora de producir (Principio de la colaboración y la cooperación), pero individualistas a la hora de distribuir y consumir (Principio del egoísmo psicológico).

Por lo tanto, la postura de Homo Economicus o agente racional, se ha enfocado a lo largo de la historia del pensamiento económico en constituir una imagen placentera del ser humano en las relaciones económicas y en brindar alternativas metodológicas de importancia para la teoría (Vidal, 2008; Roncaglia, 2017). Además, ha sido transpolada a toda la existencia humana, a sus actividades y ha marcado las relaciones del sujeto con el entorno a través del vínculo con los objetos y con otros seres vivos, incluido el propio ser humano.

Existen sociedades que se consideran destinadas a ser superiores a otras, por obra y gracia de pretenciones injustificadas del razonamiento económico (Noah, 2014). La acción colectiva de los grupos en la sociedad refleja estos criterios, justificados desde la Economía convencional que conciben al ser humano racional, superior y dueño de todos los recursos que se necesitan para que perdure la existencia de todos los seres vivos (Abitbol y Botero, 2005). 
La Economía convencional procura evidenciar y cimentar un conjunto de creencias que distorsionan el verdadero papel del ser humano, no solo hacia la sociedad, sino también hacia la naturaleza y todo el entorno que le rodea. Urge generar una visión más realista, optimista y comprometida que fomente la aparición de criterios capaces de desterrar varios mitos de superioridad y dominio del ser humano sobre todo lo que le rodea. Estas ideas pueden estar relacionadas con los siguientes argumentos:

- El entorno del ser humano es heterogéneo y está integrado por un número elevado de elementos, propiedades e interrelaciones. De esta manera, se reconoce el carácter complejo, azaroso e indeterminado del contexto, así como de la influencia que ejerce la humanidad sobre éste (Bunge, 2001).

- Los intereses y deseos que rigen las decisiones económicas de los individuos, no son totalmente dominados por el interés personal, el egoísmo y la posesión de recursos (Calvo, 2018).

- La capacidad indudablemente superior para procesar la información, sin embargo, esta continúa siendo limitada e incompleta (Tversky, A. \& Kahneman, 1971, 1974, 1983; Cortada, 2008; Kahneman, 2017).

- El individuo económico racional y maximizador de beneficios no es una máquina de tomar buenas decisiones, así como a la hora de asumir comportamientos adecuados (Roncaglia, 2017; Mullainathan y Sharif, 2013).

- Las preferencias humanas varían con el tiempo y las situaciones poseen un carácter relativo. Por tanto, son estables, aunque no estáticas (Elster, 2002; Abitbol y Botero, 2005).

Desde la perspectiva académica, existen condiciones que pueden apoyar este cambio de perspectiva. Dichas condiciones están vinculadas con el análisis tanto de la situación económica como la situación de vida de los seres humanos. Algunas de ellas podrían ser:

- Generar un debate teórico sobre el lugar (con un enfoque estructural) y el papel (desde una perspectiva funcional) que ocupa el individuo en la sociedad, a la luz del conocimiento generado desde las ciencias económicas con posiciones ajenas al enfoque convencional.

- Diversificar los enfoques investigativos. La estadística y la econometría son las herramientas, por excelencia, para la investigación en Economía convencional. Sin embargo, desde que Vernon Smith propuso la metodología del valor inducido, ha tomado fuerza la experimentación en sus dos versiones: de campo y de laboratorio.

- Fomentar el auge de la Economía experimental permite la evaluación sistemática y controlada de los principales postulados de la Economía a nivel de campo o de laboratorio. No obstante, no es posible en que materia de investigación social se pueda confirmar reiteradamente los resultados en contextos y generaciones que han cambiado.

En este artículo se comparte el criterio de que "Las ciencias sociales, en cambio, han fracasado de modo lamentable en su pretensión de mejorar las condiciones humanas" (Von Mises 1980, p. 28). Actualmente, se ha extendido una postura crítica entre los economistas de los grandes centros académicos. En este sentido, el movimiento por una Economía post-autista se inició en La Sorbona en el año 2000, cuando un grupo de estudiantes de Economía de varias universidades francesas mostraron su inconformidad por la formación que recibían. En el año 2001, decenas de estudiantes de doctorado de la Universidad de Cambridge se sumaron a la iniciativa y, posteriormente, más de 700 estudiantes de la propia universidad. En 2004, el movimiento comenzó en España (Ruíz, 2016). En 2011, un grupo de 70 estudiantes de la Universidad de Harvard abandonaron sus clases de Teoría Económica, para expresar su inconformidad con la falta de pluralidad y visión crítica en la enseñanza y la investigación económica:

Ocurre con demasiada frecuencia que profesores y estudiantes de economía son capaces de desarrollar modelos analíticos abstractos de elevada complejidad o de optimizar funciones de utilidad, beneficios y/o costes en relación a actividades económicas concretas; sin embargo, en términos generales, encuentran serias dificultades para comprender la dinámica y funcionamiento del sistema económico actual y sus mecanismos esenciales. Demasiado a menudo, la lección magistral, tal y como se plantea, no deja espacio a la reflexión. A pesar de la variedad y heterogeneidad de corrientes económicas existentes en la ciencia económica, se presenta exclusivamente una, a la que se le presupone capaz de explicar cualquier componente de la realidad según un método puramente axiomático (Ruíz, 2016, p. 11). 
La Economía convencional se ha encargado de generar doctrinas que fomenten un enfoque distorsionado del ser humano. Los límites entre lo mitológico y la realidad son apenas perceptibles para un observador con experiencia. Las ideas que rodean al ser humano, sus hazañas, sus conquistas y su desarrollo nublan la perspectiva de análisis objetivo de las falencias, las carencias y los obstáculos (Noah, 2018). La Economía convencional ha consolidado un conjunto de creencias que afectan la capacidad del ser humano para comprender la realidad. Sin embargo, con el paso del tiempo, varias voces y posturas académicas han brindado suficientes argumentos para revertir esta situación.

\section{IMPORTANCIA DE LA ECONOMÍA CONDUCTUAL EN LA CONCEPCIÓN HUMANA}

En la historia de la Economía el camino seleccionado ha puesto a los científicos frente a una encrucijada, limitado por los recursos metodológicos y los antecedentes teóricos con que contaban, más su sabia intuición y conocimientos (Bunge, 2001; Etxezarreta, 2015). La Economía Conductual es, actualmente, un campo reconocido dentro de la Economía académica ( Angrist y Pischke, 2015).

En la primera mitad del siglo XX se comienza a cuestionar el conocimiento económico tradicional, debido, principalmente, al enfoque deductivo-axiomático de las herramientas de análisis aplicadas. En este sentido, para Herbert Simon $(1955 ; 1972)$, los agentes económicos no cuentan con la capacidad suficiente para procesar grandes cantidades de información, analizarlas y comprenderlas, y de ahí surge el término racionalidad limitada. Desde ese momento, a partir de un grupo de posturas teóricas se rompe con la Economía clásica e imperante hasta entonces. Dentro de las posturas más significativas se hace referencia a lo siguiente:

- Análisis psicológico de las decisiones económicas de George Katona (1951)

- Racionalidad limitada de Hebert Simon $(1955,1972)$

- Teoría prospectiva de Tversky y Kahneman (Kahneman, 2017)

- Contabilidad mental de Richard Thaler (1985)

La Economía Conductual tiene una doble connotación: por un lado, la apreciación académica, como todo conocimiento científico que aporta a la explicación de la realidad; por otro lado, la crítica, la cual rompe con criterios inamovibles sobre el comportamiento humano, sus capacidades, sobre todo, los criterios fundamentados por la Economía convencional. Por lo tanto, no basta con razonar acerca de las nuevas categorías y conceptos, es necesario refutar criterios ya existentes. Se procura la transformación del problema fundamental de la Economía, desde la visión de la Economía Conductual: la escasez de recursos no es solo material, sino escasez racional (Mullainathan y Sharif, 2013), escasez informativa (Torrealba, 2020), escasez en las preferencias, escasez de certezas (Tyszka, 2015) frente a las necesidades siempre crecientes de la humanidad.

La Economía Conductual posee un carácter interdisciplinario, reclamado desde el paradigma de la complejidad para el estudio de las ciencias sociales en general y del comportamiento humano en particular (Allais, 1953). Como ha señalado Von Mises (1980) la teoría económica y la práctica humana se encuentran vinculadas, por lo tanto, todo conocimiento necesita integrarse para una mejor comprensión de las acciones de los seres humanos. Según Bunge (2001): "las ciencias sociales son una, no porque todas se hayan reducido a una ciencia más básica (...), sino más bien porque, en virtud de los puentes entre ellas, constituyen un sistema conceptual" (p. 43). De esta manera, al aplicar y desarrollar áreas prácticas como las finanzas conductuales (Rebollo y Vázquez, 2020) o el paternalismo libertario (Manrique, 2020), se emplean conocimientos integradores de la Economía con Psicología, Sociología y Antropología.

La Economía Conductual ha logrado ampliar, a través de la experimentación y la observación empírica, los fundamentos de ciertos axiomas que dificultan entender aún más la Economía. Actualmente, sus investigaciones han tenido una mayor relevancia en la aplicación de estos nuevos métodos y teorías (Sunstein y Thaler, 2017). No se trata de sustituir o superar la Economía convencional, sino que pretende transformar 
sus bases y sus principios sin perder el carácter científico, siendo consecuente con una visión del ser humano diferente y realista de sus condiciones (Tversky y Kahneman, 1971; 1974; 1983).

Según Camerer y Loewenstein (2004), la Economía Conductual pretende generar mejores teorías, realizar mejores predicciones y presentar mejores recomendaciones de políticas. El primer espacio donde esta rama de las ciencias económicas se ha impuesto es en la práctica, específicamente, en la explicación del comportamiento humano en la vida cotidiana. La Economía Conductual ha ganado espacios dentro del conocimiento al contestar algunas preguntas referentes al comportamiento de individuos y grupos que no podían ser explicadas utilizando el cuerpo teórico existente hasta el momento en las ciencias económicas.

La explicación de las desviaciones o anomalías frente a patrones de conducta esperados ha sido un logro significativo y ha generado un espacio para mostrar sus aportes. Lo importante es que mantiene el interés y la importancia del mercado, ya que justifica el funcionamiento desajustado de algunos de sus componentes (Tversky y Kahneman, 1983) al explicar el comportamiento del consumidor. Para ello, utiliza las heurísticas y los sesgos, así como el comportamiento del vendedor y comprador a la hora de optimizar los precios.

Según DellaVigna (2009), Vernon Smith sentó las bases de los experimentos económicos estandarizados. Si bien los experimentos de laboratorio fueron predominantes al inicio de la Economía Conductual, los experimentos de campo se han vuelto cada vez más importantes. Otro elemento que ha marcado distancia entre la Economía Conductual y la Economía Convencional es la unión a la Economía Experimental, con experimento de campo y de laboratorio para obtener hallazgos investigativos que contribuyan al desarrollo teórico y metodológico de la disciplina.

Este énfasis en la experimentación, a partir de la importancia de modelar las pautas de conducta, abre posturas para la investigación científica, amparada en los siguientes criterios:

1. El comportamiento humano no es racional todo el tiempo.

2. En cuanto a las utilidades y los beneficios, las pérdidas se sienten más que las ganancias.

3. El presente importa más que el futuro para las actividades económicas.

4. Todas las decisiones se enmarcan en un contexto creado por la influencia de otros sujetos.

La teoría económica clásica desarrolla en su modelo de mercado la condición de ceteris paribus, que significa: sin la influencia de otras variables porque todo lo demás se mantiene constante, conviene subrayar que en la Economía Conductual es una ilusión. El comportamiento humano, ya sea del consumidor, del vendedor, o de cualquier sujeto en rol de agente económico, se encuentra influenciado por una gran cantidad de variables y determinado por importantes contenidos de naturaleza subjetiva. La Economía Conductual ha ofrecido suficiente conocimiento para refutar este criterio vigente en toda la teoría económica, teoría del mercado y microeconomía (Tversky y Kahneman, 1971; 1983).

Dentro de los elementos más reconocidos dentro de la Economía Conductual se encuentran las heurísticas y los sesgos. Los más llamativos para los investigadores, según el criterio de Sunstein y Thaler (2017) han sido:

- El efecto anclaje. Es la disposición para calcular las decisiones según un punto de referencia previamente establecido, asumido o impuesto.

- La disponibilidad. Es la disposición para calcular con la información a la mano y visible, y que suele estar influenciada por eventos recientes o experiencias previas.

- La representatividad. Es la disposición para tomar decisiones basadas en la semejanza de la situación con prejuicios o ideas fundadas en sesgos de mayorías o percepciones sobre otras situaciones aparentemente similares.

- El optimismo y exceso de confianza. Es la sobreestimación común de las habilidades y probabilidades propias de lograr algunas tareas; supone la percepción personal de calcularse siempre por encima de la media.

- La sobreestimación de las pérdidas y la subestimación de las ganancias. Es la distancia en el valor que las personas les dan a pérdidas y ganancias, en tanto "es dos veces mayor la desgracia de perder algo que la alegría de ganar eso mismo” (Sunstein y Thaler, 2017, p. 51). 
- El enmarcado o framing. Es la influencia que el marco de referencia con el que se presenta una situación posee sobre las decisiones que toman las personas.

Otro componente de valor teórico, metodológico y práctico de estos autores ha sido su propuesta del paternalismo libertario (Sunstein y Thaler, 2017). Actualmente, se aplica en el diseño y aplicación de políticas públicas en muchos países y varios gobiernos han creado sus unidades de análisis del comportamiento, como es el caso de Estados Unidos y el Reino Unido. La importancia de este concepto radica en que se reconozca el apoyo hacia las decisiones humanas.

A continuación, se exponen tres ejemplos de mitos y de creencias humanas que demuestran el comportamiento amparado, en cierta medida, en el conocimiento de la Economía convencional. La existencia humana, vista desde la Antropología, estaba desprovista, en un inicio, de justificaciones a estas prácticas sustentadas en criterios de supremacías, ideologías y divisiones dentro de la especie. La Economía Conductual fundamenta, con sus avances científicos, criterios que causan debate en torno a las prácticas humanas, sus decisiones y su potencial cognitivo.

\subsection{DESMITIFICANDO LA ESPECIE HUMANA: LA ILUSIÓN DE LA HEGEMONÍA O ANTROPOCENTRISMO}

El Homo Sapiens es un ente devenido de la evolución de las especies, como se justifica en la teoría de la evolución de Charles Darwin (Otálora-Luna, Aldana y Viloria, 2017; Martínez, 2017; Rampello, 2019). Su surgimiento natural se debe a condiciones evolutivas circunstanciales que contribuyeron a la aparición de la especie. Hace 25000 años, aproximadamente, el Homo Sapiens habitaba el planeta como cualquier otra especie. Las creencias sobre la capacidad de adaptación, la fortaleza, el carácter social no son propias de este momento inicial de la evolución de la especie humana (López, 2016; Noah, 2018).

Las condiciones de vida determinaron las particularidades en la evolución del ser humano. Con el paso del tiempo, la ciencia y el conocimiento se encargaron de explicar y justificar las conductas que, de manera paulatina, iban apareciendo y perpetuaron la existencia de la especie. Las necesidades y demandas sociales fueron generando en el ser humano de la capacidad de crear símbolos para comunicarse y utilizar herramientas artificiales, lo cual permitió el desarrollo del cerebro (Tversky y Kahneman, 1971, 1974, 1983). Comienzan a construirse los mitos de superioridad, dominio y hegemonía sobre el resto de la vida en el planeta.

La Economía convencional generó en el ser humano una visión de poderío sobre los recursos naturales, olvidándose de que la especie humana es un elemento que integra la naturaleza, y como individuo, es una parte activa de la sociedad (Barrientos, 2013). El Homo Sapiens dejó de ser autárquico, para convertirse en una entidad privilegiada y depredadora de recursos: el Homo Economicus. Como se ha dicho, la presumida superioridad del ser humano favorece la aparición de determinados comportamientos que avalan la fundamentación de la racionalidad de las decisiones. Esto hace que sea aceptada tanto la domesticación como el sacrificio de animales para alimentarse, como forma de diversión u ofrenda a dioses, lo cual es uno de los mayores crímenes de la historia (Noah, 2014). El hecho de domesticar, la forma de subsistencia del ser humano se estableció hace aproximadamente 12000 años.

La Economía convencional ha legado la explicación del éxito del desarrollo humano, basada en el mantenimiento de un mayor número de personas en peores condiciones de vida (Villalobos, 2001). El aparente éxito evolutivo (gran cantidad de personas) ha sido a costa de sufrimiento individual (personas que apenas pueden sobrevivir en precarias condiciones de extrema pobreza y desigualdad económica). Basta con analizar la realidad en continentes como África y América para comprender esta verdad.

Por lo tanto, los criterios sobre la superioridad humana sobre el resto de las especies han sido fertilizados con las afirmaciones realizadas desde el conocimiento científico, extendido a través del conocimiento 
empírico y la puesta en práctica de estas ideas. El lenguaje permitió la coordinación y colaboración a gran escala para producir, pero posibilitó la trasmisión de creencias sobre una organización natural jerarquizada y presidida por la especie humana, organizada en imperios y religiones (Noah, 2014). Los mitos aumentaron la capacidad de cooperar y cohesionaron a la especie humana frente al entorno que le rodeaba.

La Economía Conductual explica que la conducta racional es solo un tipo de comportamiento del ser humano; no es una categoría superior de este, sino una singularidad donde se emplean las potencialidades cognitivas y se implica toda la esfera afectiva y volitiva del individuo en una decisión o elección. Por lo tanto, el punto de funcionamiento óptimo no es, necesariamente, donde actúe la racionalidad humana; sino donde exista una implicación de su personalidad que comprometa sus esfuerzos y que garantice el control de sus acciones futuras. Este elemento echa por tierra el carácter ilimitado de la racionalidad humana, que ha sido entendido como un elemento de superioridad sobre los demás seres vivos.

Con el descubrimiento de la presencia de heurísticas y sesgos (Kahneman, 2017) se ilustra que el ser humano presenta múltiples limitaciones cognitivas a la hora de procesar la información. Se manifiesta la existencia de determinadas trampas cognitivas que guían el funcionamiento de un sistema intuitivo para las decisiones humanas, por ejemplo, se presenta inconsistencia en las preferencias a lo largo del tiempo, aversión a la pérdida, influencias sociales, entre otras peculiaridades en el funcionamiento cognitivo del ser humano.

Por lo tanto, existe evidencia irrefutable del carácter limitado de la racionalidad humana, que aleja la idea de ser máquinas pensantes. Los seres humanos pueden afectar, con su conducta, al resto de los seres vivos, incluso, a otros individuos de su propia especie. La especie humana comete errores de manera sistemática y con poca capacidad de crítica; su actuación depende de la información que reciba de su entorno y la forma en que esta sea procesada y realiza valoraciones influenciada por elementos emocionales y subjetivos que lo alejan de la objetividad y racionalidad pura (Cortada, 2008). Estas evidencias de la Economía Conductual contribuyen a desmitificar la supuesta superioridad y hegemonía del ser humano sobre todo lo que le rodea.

\subsection{LA ILUSIÓN DE LA SINGULARIDAD: EL INDIVIDUALISMO}

En la Antigüedad el ser humano vivía en una verdadera comunidad. Prácticamente, no tenía posesiones y la convivencia era armoniosa y despreocupada. Cuando comienza a habitar en cuevas y casas, estas eran de un solo ambiente, solo paredes y techos, sin divisiones internas. No existían dominios sobre el agua, los bosques, sus frutos y los animales (Elster, 2002; Noah, 2016). Por tanto, se afirma que existía una condición para el desarrollo de la vida en la colectividad y el uso común de los recursos naturales.

Actualmente las familias destinan una habitación para cada persona. En esa habitación reproducen, prácticamente, lo mismo de la casa: baño sanitario, TV, equipos de audio, etc. Las habitaciones se convierten en hogares en miniatura, tienen puerta, ventanas, iluminación y climatización independientes. Este hecho refuerza el individualismo, ya que las personas no se preocupan por los demás, sino que tratan de mejorar su entorno de vida. El auge del individualismo ha llegado a tal punto que las personas priorizan el uso de bienes individuales antes de los colectivos (Sachs, 2008). Prevalece el uso del transporte personal, cada persona debe tener un ambiente de trabajo individual con su PC, teléfonos, impresora, fax, etc. Cada oficina debe contar con su baño personal.

La Economía convencional ha aportado a la concepción del individualismo humano al reconocer el principio del egoísmo psicológico. Se comparte el criterio donde se manifiesta que:

El egoísmo humano se ha convertido en la principal base argumental de la teoría económica convencional. Expresado como la natural propensión humana a maximizar constantemente su propio beneficio y convertido en axioma fundamental de la teoría durante los siglos XIX y XX, en tanto idea autoevidente que no requiere comprobación, este permite reducir toda la realidad comportamental del agente económico y, por consiguiente, prever todos sus movimientos y las consecuencias intencionadas o no de sus acciones. (Calvo, 2018, p. 103) 
El enfoque de Homo Economicus, propuesto por la Economía convencional es ideal y reduccionista. Las categorías de agente económico y mercado son algunos de sus mecanismos y unidades de análisis más importantes. En ambos casos, se trata de reducir las características de los grupos humanos hasta homogeneizarlas y convertirlas en elementos particulares. El concepto de individuo es empleado como forma de concreción y reducción para facilitar el estudio, sin reconocer la individualidad como elemento de diversidad que contribuye a la interpretación de las prácticas humanas.

La Economía convencional reconoce la existencia de una singularidad basada en la igualdad y semejanza, donde se concibe la estructuración del contenido subjetivo a la hora de regular la conducta y tomar decisiones de manera uniforme en todos los seres humanos; por lo que las determinantes de la conducta estarán influenciadas por la combinación particular de las condiciones externas e internas, asumiendo las características propias para el procesamiento de la información y la toma de decisiones.

La Economía Conductual brinda una perspectiva de análisis distinta y favorecida con el criterio acerca de la existencia de sesgos relacionados con las preferencias sociales. En las decisiones el individuo se preocupa por el criterio que se formen los demás de él, así como también se ve impulsado considerar la conducta de otros individuos significativos ante situaciones similares. Desde la Economía Conductual no se desestima el papel del grupo, ni se reduce a características que permitan su generalización, mas bien se fomenta el reconocimiento de las diferencias a la hora de decidir y la influencia de los demás en la elección que realizan los sujetos. Estos argumentos permiten reconsiderar los criterios que proponen la existencia singular y egoísta de los seres humanos, impulsados por el beneficio propio. Además, aportan elementos que contribuyen a considerar la diversidad y la particularidad del ser humano y sus decisiones, donde es importante la influencia de su conducta en los demás componentes sociales y naturales que le rodean.

\subsection{LAS CREENCIAS SOBRE EL GÉNERO Y LAS DIFERENCIAS SEXUALES}

El sexo, como categoría biológica, es quien divide a los machos de las hembras, con sus características hormonales y órganos sexuales, ajenos a la voluntad de los individuos. Es el enfoque de género el que asume el rol cultural de asignar a cada persona la definición, según la orientación que desee. Un ejemplo ilustrativo de este fenómeno ocurre en la medicina y en sus distintas especialidades. Los pediatras no se diferencian por el sexo ni género de los niños; el cardiólogo es capaz de operar el corazón de hombres y mujeres por igual, el ortopédico analiza sistema óseo sin importar el sexo. Solo existen dos especialidades separadas por el sexo: el ginecólogo (atiende el sistema reproductor femenino: útero, vagina y ovarios) y el urólogo (se especializa el sistema reproductor masculino, aunque también analiza las patologías del sistema renal de ambos sexos).

Ahora bien, a pesar de los elementos biológicos que confirman que son dos clases de individuos de la misma especie (por tanto, comparten el genoma y muchas características más, aunque de sexos diferentes), múltiples investigaciones psicológicas establecen diferenciación entre competencias, habilidades, capacidades, afectos, etc., de cada uno de ellos. La manifestación de este hecho depende más de lo histórico, cultural y social, que de condiciones psicológicas y biológicas de los individuos.

La educación, asumida como el proceso cultural de socialización y formación de la personalidad, delimita claramente las características de cada género, enseña el comportamiento de mujeres y hombres, instruye los roles, funciones, características, deberes y derechos, y todos los demás mitos asociados con el género. El papel asignado al género es trasmitido y debe ser interiorizado; la sociedad valora el desempeño del individuo dentro del rol de género que le corresponde, creando las condiciones para el funcionamiento del arquetipo asociado al género.

Dentro de los mitos que la Economía convencional se ha encargado de consolidar con relación al género se encuentra el hecho de asociar el poder al género. Este justificó las diferencias entre hombres y mujeres desde varias posturas. Uno de ellos, quizás el más visible para todos, es el papel del individuo de sexo masculino al frente de las grandes instituciones y el dominio de las enormes fortunas mundiales. Resulta muy 
frecuente encontrar a hombres dirigiendo la mayoría de las instituciones internacionales, los gobiernos de la mayoría de los países, los destinos de los organismos económicos multilaterales. Se ha trasmitido el criterio desde la Economía convencional, de que los varones tienen mejores resultados en funciones de dirección y administración que las mujeres. También, la Economía convencional justificó y defiende aún la diferencia de salarios entre hombres y mujeres (Elster, 2002; Cabezas, 2018; Jabbaz, Samper-Gras y Díaz, 2019; Vargas y Huertas, 2019).

Otro elemento ilustrativo de este fenómeno es la ausencia de investigadoras mujeres dentro de los libros clásicos de Economía: no aparecen en los manuales ni dentro de las referencias bibliográficas de los libros de textos de Introducción a la Economía. La escasez de mujeres reconocidas en la Economía por el aporte de su intelecto, es un elemento significativo que llama la atención de los estudiantes, ya que la desproporción es muy grande. El pensamiento económico construido por las mujeres ha sido marginado dentro de los grandes centros de generación de conocimiento económico.

Unido a ello se aprecia el hecho de la poca representatividad de las mujeres en los Premios Nobel de Economía. Solo dos mujeres han recibido este reconocimiento, siempre acompañadas de hombres: la estadounidense Elinor Ostrom en 2009 y la francesa Esther Duflo en 2019 (Roux, 2006; Almansa, 2020). Esta cantidad representa el 2,4\% del total de premios Nobel de Economía entregados a lo largo de la historia. Sin lugar a duda, otra evidencia de las diferencias entre hombres y mujeres dentro de la Economía.

La Economía Conductual no considera al hombre más racional que la mujer, o viceversa; es más supone la capacidad de todos los seres humanos de decidir bajo la influencia de las heurísticas y los sesgos. En ninguna de las explicaciones a las distintas conductas lo hace enfocado en el género, lo que demuestra que las capacidades y habilidades entre hombres y mujeres no son posibles de diferenciar de manera estandarizada. Estas ideas contribuyen a derrumbar la hegemónica posición del hombre frente a la mujer, presente en la mayoría de las culturas caracterizadas por el machismo y que la Economía convencional se encargó de fomentar.

\section{CONCLUSIONES}

Múltiples problemas afectan la existencia del ser humano en la Tierra. La solución a los principales problemas que enfrenta la humanidad depende de la toma de conciencia y el cambio de conducta humana. Para ello es necesario concebir, desde una perspectiva interdisciplinaria, activa y transformadora a los seres humanos. Esa es la propuesta teórica fundamental que se sustenta en esta investigación.

La Economía convencional ha justificado el uso irracional de los recursos, la desigual distribución de la riqueza y el desproporcionado crecimiento y desarrollo económico en determinadas regiones. Lo anterior contribuye a la formación de mitos sobre la superioridad y hegemonía de la especie humana y de sus capacidades cognitivas. Se ha consolidado el supuesto de un ser humano hegemónico sobre los demás seres vivos, individualista dentro de su propia sociedad y diferenciado entre hombres y mujeres. Todo esto amparado en la concepción de Homo Economicus, que enfatiza en la racionalidad ilimitada del ser humano.

Frente a esta posición de la Economía convencional, surge una perspectiva más integradora, práctica, sensible y realista: la Economía Conductual; esta se encarga de demostrar que la racionalidad humana es limitada, la información se percibe de manera parcial, se analiza de modo incompleto y las decisiones están influenciadas por los heurísticos y sesgos cognitivos, además de las emociones y estados de ánimo de las personas. El sentido que encierra esta idea es el cimiento de diversos cuestionamientos sobre algunos mitos sobre el ser humano, su influencia sobre los demás seres vivos y su relación con otros seres humanos. Por tanto, existe evidencia que desmitifica la superioridad, singularidad y diferenciación basada en el género en la especie humana. 


\section{REFERENCIAS BIBLIOGRÁFICAS}

Abitbol, P. y Botero, F. (2005). Teoría de elección racional: estructura conceptual y evolución reciente. Colombia Internacional, 62, 132-145. https://doi.org/10.7440/colombiaint62.2005.08

Allais, M. (1953). Le Comportement de L'Homme Rationnel devant Le Risque: Critique des Postulats et Axiomes de L'Ecole Americaine. Econometrica, 21 (4), 503-546. https://doi.org/10.2307/1907921

Almansa, R. M. (2020). Mujeres y meritocracia. Raíces históricas de un paradigma y posibilidades actuales de transformación. El Futuro del Pasado, 11, 593-635. https://doi.org/10.14516/fdp.2020.011.020.

Angrist, J. y Pischke J.S. (2015). Mastering'Metrics: the Path from Cause to Effect. Princenton University Press.

Ávila-García, P. (2016). Hacia una ecología política del agua en Latinoamérica. Revista de Estudios Sociales, 55, 18-31. http://dx.doi.org/10.7440/res55.2016.01.

Barrientos, J. (2013). Peter Singer: senderos para un giro copernicano ético. Visión Libros.

Bentham, J. (1948). An introduction to the principles of morals and legislation. Hafner Pub. Co.

Bunge, M. (2001). La relación entre la sociología y la filosofía (2da ed.). Edaf, S.A.

Cabezas, E. (2018). Mayores Oportunidades y también Mayores Esfuerzos. Un Enfoque de Género de las Desigualdades Remunerativas para la Economía Ecuatoriana entre 2006 y 2014. Revista Cuestiones Económicas, 28(2), 13-48. https://bit.ly/3ofldFx.

Calvo, P. (2018). Cuestiones éticas y emocionales alrededor del egoísmo económico. Investigación Económica, 77(304), 102-134. https://bit.ly/3krCREL.

Camerer, C. y Loewenstein, G. (2004). Behavioral Economics: Past, Present and Future. En C. Camerer, G. Loewenstein y M. Rabin (Eds.), Advances in Behavioral Economics (pp. 3-5). Princeton University Press.

Cejudo, R. (2019). Utilitarismo. Telos: Revista Iberoamericana de Estudios Utilitaristas, 23(1-2), 53-65. https://doi. org/10.15304/t.23.1-2.6431

Cortada, N. (2008). Los sesgos cognitivos en la toma de decisiones. International Journal of Psychological Research, I(1), 68-73. https://doi.org/10.21500/20112084.968

DellaVigna, S. (2009). Psychology and Economics: Evidence from the Field. Journal of Economic Literature, 47(2), 315-372. 10.1257/jel.47.2.315

Elster, J. (2002). Alquimias de la mente. La racionalidad y las emociones. Paidós.

Etxezarreta, M. (2015).¿Para qué sirve realmente la Economía? Paidós.

García-Andrés, G. (2016). Por un cambio en la economia: La revolución necesaria. RBA Libros.

García-Rubio, M. (2016). El objeto de estudio de la economía. Un enfoque crítico en perspectiva histórica. En F. García y A. Ruíz (Eds.). Hacia una economia más justa. Una introducción a la economía crítica, (pp. 25-48). Economistas sin Fronteras.

Hernández, R., Fernández, C. y Baptista, M.P. (2014). Metodología de la Investigación. Sexta Edición. McGraw-Hill / Interamericana Editores, S.A. de C.V.

Jabbaz, M., Samper-Gras, T. y Díaz, C. (2019). La brecha salarial de género en las instituciones científicas. Estudio de caso. Convergencia, 26(80), 1-27. https://doi.org/10.29101/crcs.v26i80.11248.

Katona, G. (1951). Psychological Analysis of Economic Behavior. McGraw-Hill.

Kahneman, D. (2017). Pensar rápido, pensar despacio. Debolsillo.

López, M. (2016). La extensión tripartita de la teoría dual de razonamiento y sus repercusiones para las investigaciones sobre el autismo. Nóesis, 25(50), 245-262. http://dx.doi.org/10.20983/noesis.2016.2.10

Manrique, H. (2020). La toma de decisiones: entre la intuición y la deliberación. Editorial EAFIT, Universidad del Norte.

Martínez, M. (2017). Implicaciones de la teoría de la evolución en la filosofía. Metatheoria-Revista de Filosofía e Historia de la Ciencia, 8(1), 13-29. https://bit.ly/3kyBO5Q. 
Dr. Armenio Pérez Martínez, et al. Desmitificando al ser humano desde la Economía Conductual

Miller, L. M. (2006). Experimentos de orientación teórica. Una discusión metodológica. EMPIRIA. Revista de Metodologia de Ciencias Sociales, 12, 89-110. https://www.redalyc.org/pdf/2971/297124008004.pdf

Mullainathan, S. y Sharif, E. (2013). Scarcity: Why Having too Little Means so Much. Allen Lane.

Noah, Y. (2014). Sapiens. De animales a dioses: Una breve historia de la humanidad. Debate.

Noah, Y. (2016). Homo Deus: Breve historia del mañana. Debate.

Noah, Y. (2018). 21 lecciones para el siglo XXI. Debate.

Otálora-Luna, F., Aldana, E. y Viloria, A. (2017). Crítica a la teoría de la evolución pura: hacia la belleza estructural. LudusVitalis, 25(47), 167-185. https://bit.ly/35v1jly.

OXFAM Internacional (2021, 25 de enero). El virus de la desigualdad. Cómo recomponer un mundo devastado por el coronavirus a través de una economia equitativa, justa y sostenible. OXFAM Internacional.org. https://www.oxf am.org/es/informes/el-virus-de-la-desigualdad

Paz, M. (2016). Redes trasnacionales de organizaciones indígenas. Análisis del uso de las redes en conflictos socioambientales. Revista de Estudios Sociales, 55, 62-72. http://dx.doi.org/10.7440/res55.2016.04.

Pérez, A.y Rodríguez, A. (2020). Pensar la Economía: contribuciones humanistas para un nuevo conocimiento. Revista Economia Coyuntural, Revista de temas de perspectiva y coyunturas, 5(3), 125-146. https://bit.ly/3jgQXHu

Quintana, G. H. y Galarza, D. R. (2017). La Economía, lo social que miramos y la ciencia que enseñamos. Revista Experiencias. 1(1), 24-28. https://bit.ly/3tIWb50

Rampello, S. (2019). Los sesgos en la toma de decisiones. Revista Perspectivas de las Ciencias Económicas y Jurídicas, 9(1), 85-94. http://dx.doi.org/10.19137/perspectivas-2019-v9n1a06

Rebollo, D. y Vázquez, X. (2020). La heterogeneidad de los agentes inversores en las series de la Bolsa Mexicana de Valores. EconoQuantum, 17(1), 69-94. https://doi.org/10.18381/eq.v17i1.7095.

Roncaglia, A. (2017). Breve historia del pensamiento económico. Prensas de la Universidad de Zaragoza.

Ros, J. (2019). Los retos de la política económica. ECONOMÍAunam, 16(46), 16-23. https://bit.ly/36xL5He.

Roux, D. (2006). Los premios Nobel de Economía. Ediciones AKAL, S.A.

Ruíz, A. (2016). Introducción a la Economía Crítica. Un apunte crítico sobre los contenidos y los manuales de economía. En F. García y A. Ruíz (Eds.). Hacia una economía más justa. Una introducción a la economía crítica, (pp. 7-25). Economistas sin Fronteras.

Sachs, J. (2008). Economia para un planeta abarrotado. Debate.

Simon, H. (1955). A Behavioral Model of Rational Choice. The Quarterly Journal of Economics, 69(1), 99-118.

Simon, H. (1972). Theories of Bounded Rationality. En C. McGuire y R. Redner (Eds.), Decisions and Organization, (pp. 161-176). North-Holland Publishing Co.

Sunstein, C. y Thaler, R. (2017). Un pequeño empujón: el impulso que necesitas para tomar mejores decisiones sobre salud, dinero y felicidad. Taurus.

Taylor, S.J. y Bodgan, R. (1987). Introducción a los métodos cualitativos de investigación. La búsqueda de significados. Paidós Ibérica S.A.

Thaler, R. (1985). Mental Accounting and Consumer Choice. Marketing Science, 4(3), 199-214. https://bit.ly/3oze YxB.

Tirole, J. (2017). La Economía del bien común. Penguin Random House Grupo Editorial.

Torrealba, M. (2020). Consideraciones sobre las condiciones para el ejercicio del fact checking en Venezuela en tiempos de pandemia. Temas de Comunicación, 40, 149-158. https://bit.ly/3nRoxJP

Tversky, A. y Kahneman, D. (1971). Belief in the law of small numbers. Psychological Bulletin, 76(2), 105-110. htt ps://doi.org/10.1037/h0031322

Tversky, A. y Kahneman, D. (1974). Judgment under Uncertainty: Heuristics and Biases: Biases in judgments reveal some heuristics of thinking under uncertainty. Science, New Series, 185(4157), 1124-1131. https://www.scien ce.org/doi/10.1126/science.185.4157.1124 
Tversky, A. y Kahneman, D. (1983). Extensional versus intuitive reasoning: The conjunction fallacy in probability judgment. Psychological Review, 90(4), 293-315. https://doi.org/10.1037/0033-295X.90.4.293

Tyszka, T. (2015). Ambiguity Aversion. En M. Altman (Ed.), Real-World Decision Making: an Encyclopedia of Behavioral Economics, (pp. 12-14). Greenwood.

Vargas, E. y Huertas, C. (2019). Reflexión sobre la precariedad laboral y sus consecuencias desde un enfoque de género. Revista Interamericana de Psicología Ocupacional, 38 (2), 104-114. http://revista.cincel.com.co/index.php/RP $\mathrm{O} /$ article/view/243/pdf

Vidal, G. (2008). La Teoría de la Elección Racional en las Ciencias Sociales. Sociológica, 23(67), 221-236. http://ww w.scielo.org.mx/pdf/soc/v23n67/v23n67a9.pdf

Villalobos, J. V., Ramírez, R. I. y Díaz-Cid, L. (2019). Bioética y biopoder: perspectivas para una praxis pedagógica desde la ética de Álvaro Márquez-Fernández. Utopía y Praxis Latinoamericana, 24(87), 65-77. http://doi.org/ 10.5281/zenodo.3463783.

Villalobos, J.V. (2001). Derecho, racionalidad y supuesto metodológico de la Modernidad. Utopía y Praxis Latinoamericana, 6(12), 64-82. https://www.redalyc.org/pdf/279/27901206.pdf.

Von Mises. L. (1980). La Acción Humana. Tratado de Economía (4ta edición). Unión Editorial S.A. https://bit.ly/ 3rORMvw 